\title{
Graphene based Web Framework for Energy Efficient IoT Applications
}

\author{
Dr. Joy Iong Zong Chen, \\ Professor, \\ Department of Electrical Engineering, \\ Da-Yeh University, \\ Taiwan. \\ Email id: jchen@mail.dyu.edu.tw
}

\section{Lu-Tsou Yeh,}

Department of Electrical Engineering,

Da-Yeh University,

Taiwan.

\begin{abstract}
Energy efficiency is a crucial factor to be considered while designing sensor network based Internet of Things (IoT) applications due to the compromise in terms of power constraint in these devices. The overall network and IoT device lifetime may be increased significantly by enhancing the energy conservation schemes. Design changes, duty cycle optimization at the MAC layer and various other solutions are proposed to achieve efficiency in energy utilization. In IoT sensing applications based on cloud computing, these challenges are overcome by means of integrating the constrained application mechanism with abstraction layer as proposed in this paper. The round trips for graph-based data representation and data delivery are reduced by incorporating the cloud services with an efficient web framework in order to achieve efficient data management and energy conservation in the IoT sensing applications. In specific applications, the aforementioned objectives may be achieved by the integration of potential technologies. Google cloud services along with Graphene web framework, RIOT OS and Z1 IoT motes are used for developing a testbed in the constrained applications at the abstraction layer for interfacing the proposed implementation. The sensor networks in IoT applications have achieved significant reduction in energy consumption and delay of data delivery using the proposed method when compared to the existing baseline approaches.
\end{abstract}

Keywords: Wireless sensor networks, Internet of things, Abstraction layer, Graphene 
Journal of Information Technology and Digital World (2021)

Vol. 03/ No. 01

Pages: $18-28$

https://www.irojournals.com/itdw/

DOI: https://doi.org/10.36548/jitdw.2021.1.003

\section{Introduction}

The Internet of Things (IoT) applications on a large scale are realized by establishing modern cloud services, next-generation wireless networks and innovative sensor modules [1]. The communication networks are used for transmitting the data collected by interacting with the environment by the sensing abilities of the IoT devices [2]. There is a rapid increase in the total sensing modules linked to the internet and is expected to reach over 500 billion by 2030 . In case of massive interaction and sensing applications, the existing sensor network technologies and standards are insufficient. This causes hindrance towards large scale development of smart IoT applications [3]. Overly connected services are provided by the huge range of IoT sensing applications equipped with sensing platforms, computation and communication with the rapid evolution of modern technologies. With the increase in the number of modern applications, the count of participating sensor devices has been multiplying steadily leading to the increase in the IoT network energy demands [4]. Machine-to-machine communication between IoT devices with interconnection between over 12.3 billion mobile devices is expected by the year 2022 according to an estimation. Without the need for recharging of batteries or device replacement, the network life-time may be extended by implementing energy efficient function in the IoT platforms that are battery operated [5].

Voluminous and continuous data is generated by IoT sensing applications where bottlenecks may be caused by the constrained sensor modules during energy consuming operations and data communication [6]. Multimedia applications with dense sensor networks and IoT networks are some of the examples of such applications. For transferring multimedia data such as images, video or audio from the physical environment, dense deployment of devices in the wireless sensor networks connected via internet are used [7]. The proposed data and energy management model for IoT sensing application uses constrained application protocol based cloud services while interfacing efficient web framework. Evaluation of this model is performed with respect to target applications by implementing a testbed with real time IoT devices. 
Journal of Information Technology and Digital World (2021)

Vol. 03/ No. 01

Pages: $18-28$

https://www.irojournals.com/itdw/

DOI: https://doi.org/10.36548/jitdw.2021.1.003

\section{Related Works}

Netflix introduced technologies such as Falcor and GraphQL as a solution to decrease bandwidth utilization in its application. Connected nature of data is used for reducing the generation of traffic during this process [8]. In cloud IoT networks based on RIOT, for sensing application scenarios, the approaches presented in this paper are based and incorporated. Several energy utilization optimization schemes that exist make use of duty cycling enhancement. This is avoided by utilizing the IoT applications with an abstraction layer [9]. The complexity and semantics of the Graphene language, its properties and utilization are analyzed. For robust web interfaces, delay improvements are obtained along with the computation for internet scenario, that enables handling large volume of Graphene [10]. In order to achieve high levels of interoperability in complex data-driven ecosystems, REST APIs are used. In various segments of the ecosystem, the Graphene benefits are achieved in the main API while leveraging certain benefits in the protocol [11]. Linked data consumption is elevated for application development in linked open data clouded by a linked data querying scheme called Graphene-LD [12].

Power-aware applications may be used by the implementation of data communication reduction schemes for decreasing energy utilization [13]. The queries may be combined for multiple resources into a single endpoint by means of the abstraction layer for further reduction of data traffic. The duty cycling mechanism may be optimized by emphasizing the reduction of sensor device based active slots. In low power wake-up radio, the networking and hardware details are studied by certain researchers. While targeting applications with high data rate, the duty cycling scheme may be compromised to a certain extent [7]. For optimization of data transaction, the application layer may be incorporated to achieve delay minimization and power conservation [14]. The performance of the model may be enhanced by transforming data from the application layer protocol with the help of gateway as a service. The server layers and graph-based querying abstraction layers are integrated at the application level with the help of constrained application protocol (CoAP) [15]. Certain research works introduced the implementation of IoT in application layer protocols. Firebase and other services may be used 
Journal of Information Technology and Digital World (2021)

Vol. 03/ No. 01

Pages: $18-28$

https://www.irojournals.com/itdw/

DOI: https://doi.org/10.36548/jitdw.2021.1.003

for HTTP based internet communication and CoAP IoT protocol mapping as emphasized in these work.

\section{Proposed Work}

The communication sub-system consumes over $60 \%$ of the nodal energy according to a study. In the dry battery powered IoT devices, energy is a highly scarce resource. There is an increase in the number of active data transmission nodes with the increase in the rate of data transmission leading to an increase in the packet losses and collision during data transmission due to the shared wireless channel. The node level transmissions may be reduced to address these challenges. This enables reduction of energy consumption and data transmission collision due to the reduced transmission results. This further avoids packet retransmission and the energy wastage caused by it. The energy efficiency challenge is addressed by the proposed architecture as represented in figure 1.

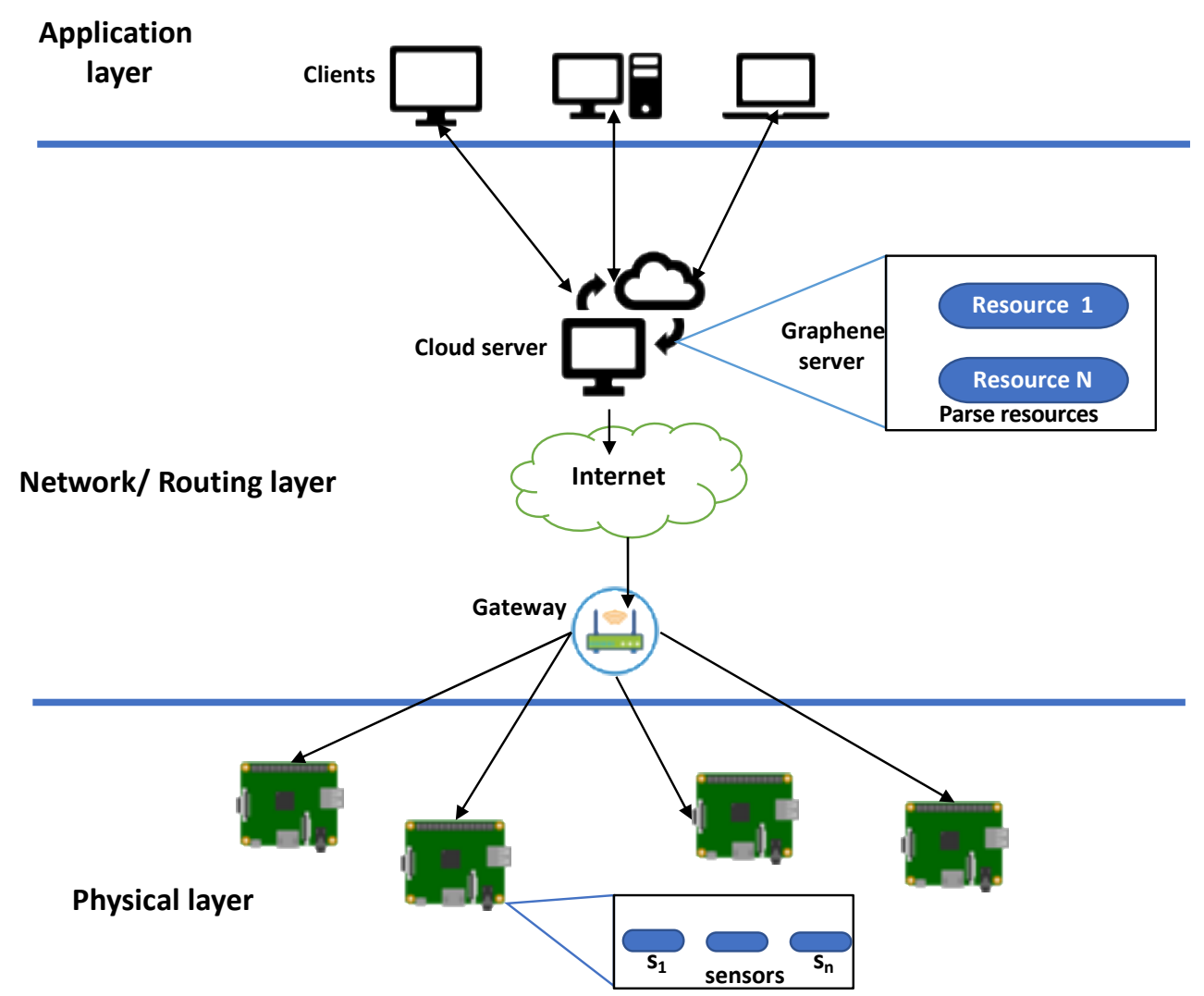

Fig. 1. Proposed Architecture in a layered setup 
Journal of Information Technology and Digital World (2021)

Vol. 03/ No. 01

Pages: $18-28$

https://www.irojournals.com/itdw/

DOI: https://doi.org/10.36548/jitdw.2021.1.003

Integration of Graphene framework with the sensing applications with constrained IoT libraries is independent of implementation due to several prominent features. In IoT applications, the communication delay is reduced by increasing the bandwidth utilization and energy efficiency with the help of this model. The constrained application protocol, cloud services and abstraction layer are integrated with IoT in the proposed model. Data management and efficient energy utilization is achieved by integration of cloud services with IoT CoAP protocol on the server and client modules as well as the abstraction layer of Graphene. Various data injection challenges are overcome by evaluation of the proposed model under multiple scenarios by implementing a testbed for IoT applications with cloud or web technologies.

A flexible and scalable paradigm is obtained while representing IoT data in queries notion and graphs using the Graphene abstraction layer in the proposed model. The changes and evolution are simplified by performing modification of data in the form of graphs. Any required query may be designed by the IoT clients with great flexibility while implementing the client side graph query module. Hence, for all possible data requests, implementation of end points by the server is not required. No extra data is bundled in the object while achieving the required data transaction in a declarative manner while specifying the data exchange requirement at the client sensor layer.

\section{Results and Discussion}

Communication optimization is performed for achieving energy efficiency at the overall network while prolonging the IoT device operation lifetime with the proposed approach. Sparse deployment of current IoT networks and expected dense deployment of future IoT networks are found to be effective on implementation of the proposed approach. Significant energy efficiency improvement is observed from the experimental results in which 5 IoT devices are contained in a testbed environment under a single site. Further exploration and evaluation of the proposed idea may be performed with larger number of IoT devices in a bigger experimental setup based on the obtained results. 
Journal of Information Technology and Digital World (2021)

Vol. 03/ No. 01

Pages: $18-28$

https://www.irojournals.com/itdw/

DOI: https://doi.org/10.36548/jitdw.2021.1.003

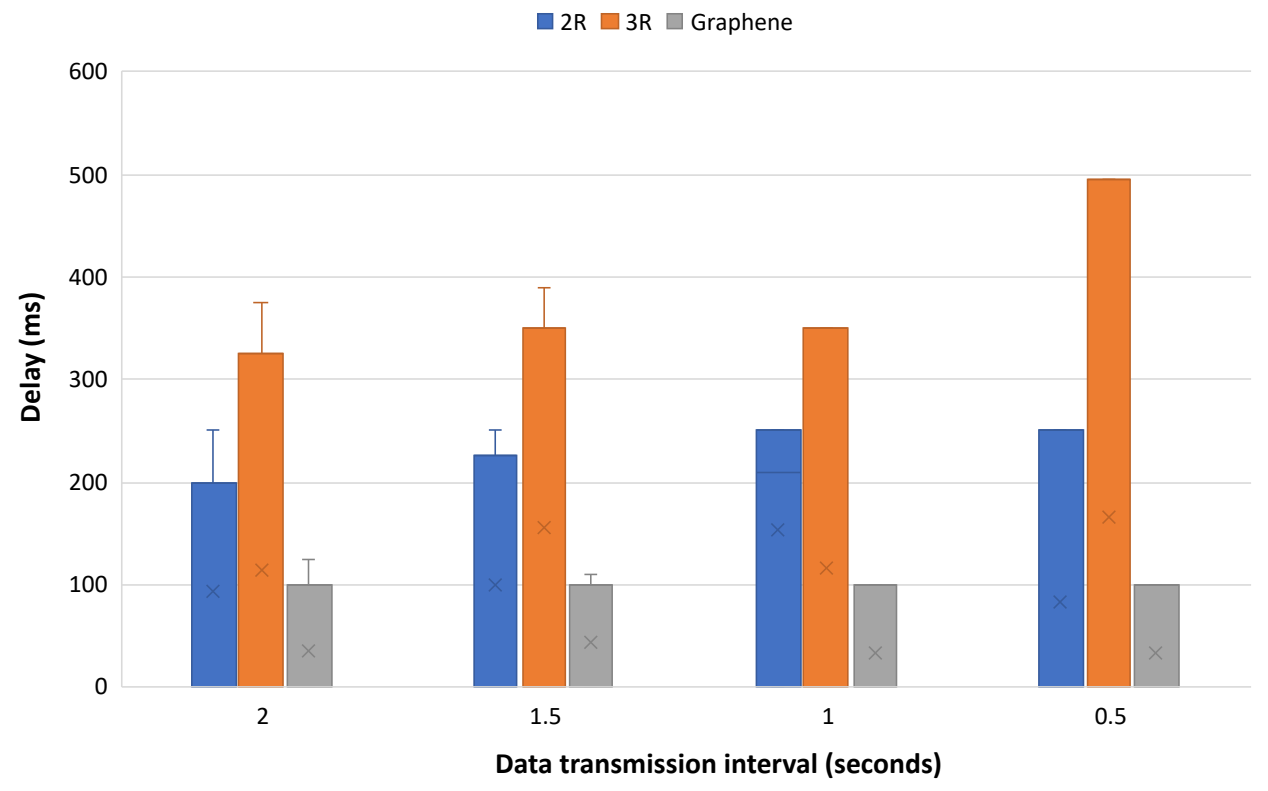

Fig. 2. Comparison of average delay with respect to data transmission interval

For all data transmission intervals, the REST-based schemes offer considerable increase in energy consumption. With further increase in the data transmission rate, the energy consumption also increases. Less than half of the energy cost is used for achieving data delivery by the proposed scheme that outperforms the existing baseline REST based models. The device based evaluation scenario does not have throughput variations or significant packet loss, due to which the throughput results and Packet Delivery Ratio (PDR) are insignificant for this scenario. The proposed Graphene based scheme offers improved performance confidence for both metrics as represented by the error bars and spread of values. 
Journal of Information Technology and Digital World (2021)

Vol. 03/ No. 01

Pages: $18-28$

https://www.irojournals.com/itdw/

DOI: https://doi.org/10.36548/jitdw.2021.1.003

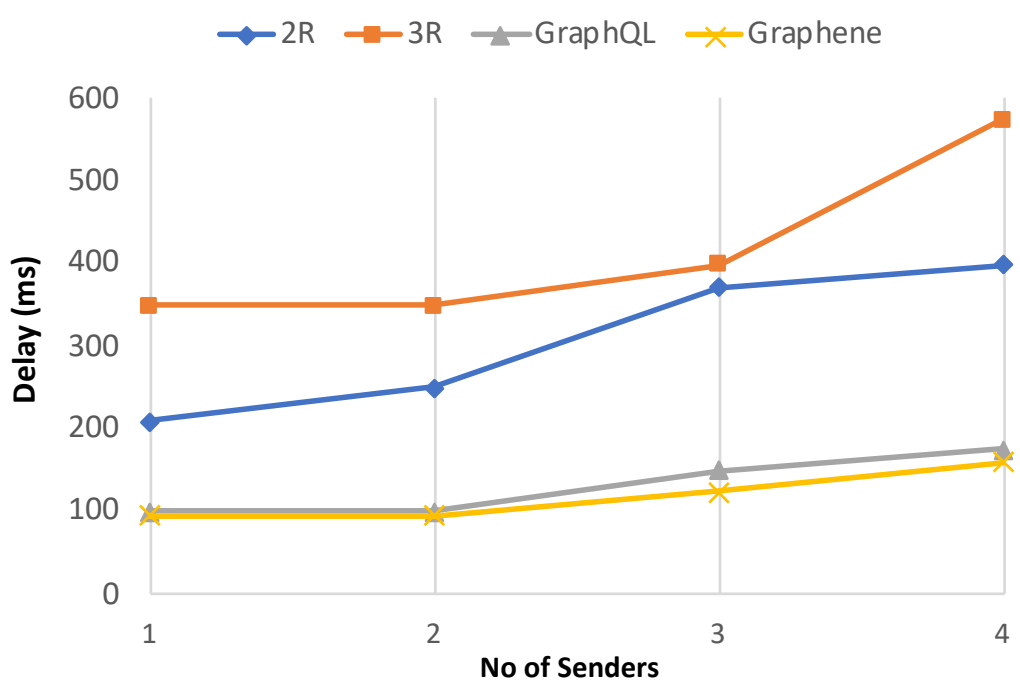

Fig. 3. Comparison of average delay for multiple senders under various network configurations

The device configurations are adjusted to form a dumbbell-shaped network topology with diverse number of IoT client devices in which direct single-hop connectivity is provided to the cloud gateway or border router from all the senders or devices. Multiple active senders based on the number of devices connected in the network increases the data load in the network. Comparison of performance is done under various settings such as data transmission to three cloud resources, data transmission to CoAP server, data registering to resources with distinct endpoints and do on. The comparison of system throughput and packet delivery ratio based on successful data transfer is as shown in figure 3 and 4 respectively. The area containing the border nodes and transmission devices in the topology is the same in this model. The REST architecture performance is compared with the performance of the proposed model. The proposed model offers promising performance when compared to the existing schemes. 
Journal of Information Technology and Digital World (2021)

Vol. 03/ No. 01

Pages: $18-28$

https://www.irojournals.com/itdw/

DOI: https://doi.org/10.36548/jitdw.2021.1.003

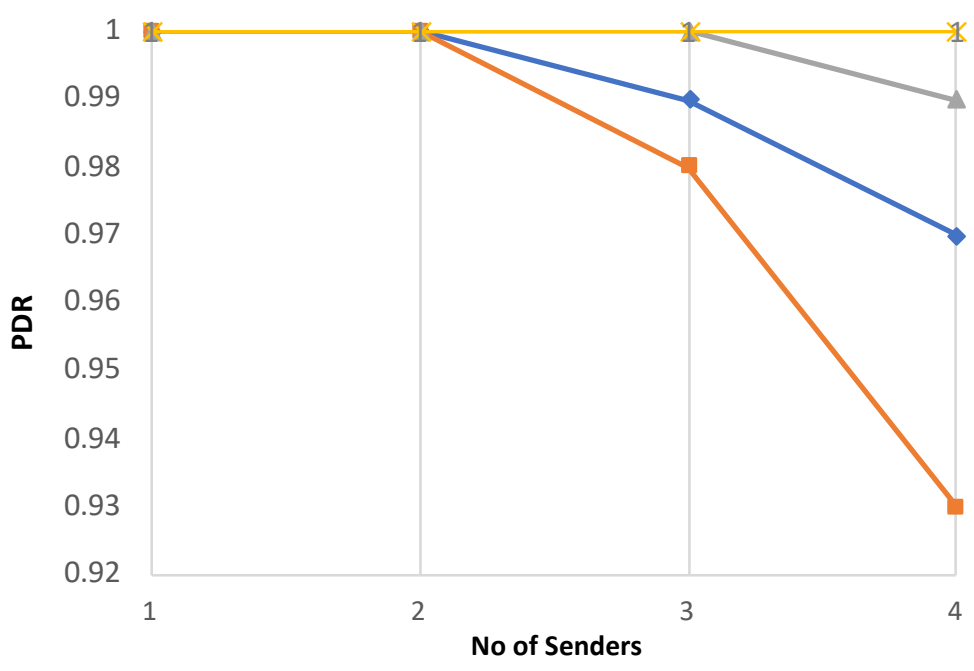

Fig. 4. Comparison of Packet delivery ratio (PDR) for multiple senders under various network configurations

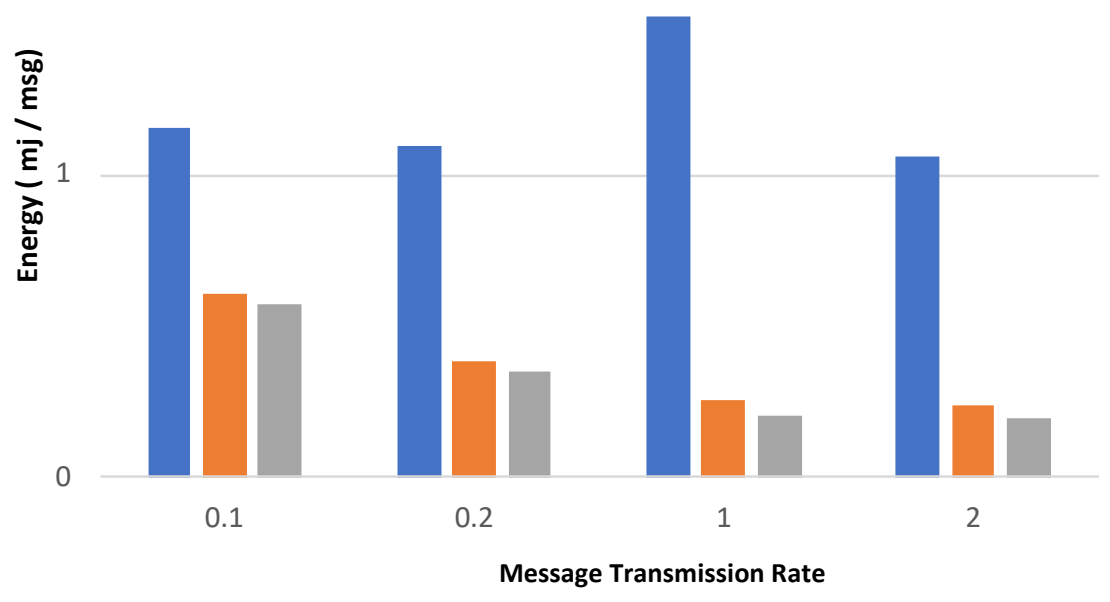

Fig. 5. Comparison of energy with increasing transmission rate

In a home or indoor environment, the topologies are positioned such that the receiver and sender gateway are placed at a distance of $12 \mathrm{~m}$ for performing the connectivity experiments. Direct connectivity is restricted by setting low power transmission value during the first 
Journal of Information Technology and Digital World (2021)

Vol. 03/ No. 01

Pages: $18-28$

https://www.irojournals.com/itdw/

DOI: https://doi.org/10.36548/jitdw.2021.1.003

scenario. Intermediate forwarders are formed by placing two motes of same transmission power at equal distance for providing data communication connectivity. Multi-hop path scheme is used for communication is performed to the next forwarder based on the connectivity range of each mote. The transmission power is increased while the intermediate motes are switched off in another scenario. This provides a direct connectivity between the gateway node and the infiled sensing server. The transmission rate and interval between the synchronous request messages are varied for multiple cycles. For every request-response message exchange, the average energy consumption and average round trip delay is measured. For every requestresponse exchange, at specific receiver gateway per unit time, the average response receiving rate is attained and duty cycling is performed. Figure 5 provides the comparison of energy consumption of various experimental setup. Connectivity provided between the transmitter and the receiver is the major variation in the transmission power.

\section{Conclusion}

The drawbacks of traditional communication protocols such as the constrained nature of the devices impose several challenges in applications involving IoT sensing on large scale realization. In web-based linked data, the communication efficiency challenges are resolved with the introduction of an application layer framework termed Graphene. The IoT application scenarios based on sensor networks are applied with the abstraction layer on leveraging the appropriate features. In RIOT OS, the real IoT motes are incorporated and their performance is analyzed under various experimental scenarios and applications that require continuous transmission of IoT sensing data. The transaction delay and energy utilization are reduced significantly for the target cases on validation on the Graphene based abstraction layer by implementing the proposed approach according to the empirical studies. A high power direct communication proposal is tested during multi-hop IoT communication in hierarchical structure considering to relieve the energy-wave problem. The experimental setup offered improved performance under this scenario. Thus the modern IoT applications may be integrated with modern web software for efficient utilization. Future scope is focused on strengthening the model by implementation on larger IoT networks and application of modern technology. 
Journal of Information Technology and Digital World (2021)

Vol. 03/ No. 01

Pages: $18-28$

https://www.irojournals.com/itdw/

DOI: https://doi.org/10.36548/jitdw.2021.1.003

\section{References}

[1] Sathyamoorthy, P., Ngai, E. C. H., Hu, X., \& Leung, V. (2017). Profiling energy efficiency and data communications for mobile internet of things. Wireless Communications and Mobile Computing, 2017.

[2] Hietala, J. (2020). Real-time two-way data transfer with a digital twin via web interface.

[3] Rasool, S., Khan, R., \& Mian, A. N. (2019). GraphQL and DC-WSN-based cloud of things. IT Professional, 21(1), 59-66.

[4] Sultanov, M. M., Gorban, Y. A., Smirnov, A. A., \& Yurov, V. A. (2021, March). Development of a centralized system for data storage and processing on operation modes and reliability indicators of power equipment. In 2021 3rd International Youth Conference on Radio Electronics, Electrical and Power Engineering (REEPE) (pp. 15). IEEE.

[5] Pružinec, F., Mego, P., \& Đuračiová, R. (2020). A low-cost web-based smart street lighting geographical information system for smaller towns and villages. Open Computer Science, 10(1), 450-458.

[6] Iggena, T., Bin Ilyas, E., Fischer, M., Tönjes, R., Elsaleh, T., Rezvani, R., ... \& Holmgård Christophersen, S. (2021). IoTCrawler: Challenges and Solutions for Searching the Internet of Things. Sensors, 21(5), 1559.

[7] Shahinmoghadam, M., \& Motamedi, A. (2020, August). An ontology-based mediation framework for integrating federated sources of BIM and IoT data. In International Conference on Computing in Civil and Building Engineering (pp. 907-923). Springer, Cham.

[8] Juuti, J. (2020). Definition and implementation of general-purpose IoT cloud backend.

[9] Xie, C., Yu, B., Zeng, Z., Yang, Y., \& Liu, Q. (2020). Multi-layer internet of things middleware based on knowledge graph. IEEE Internet of Things Journal.

[10] Jukić, O., Heđi, I., \& Ciriković, E. IoT cloud-based services in network management solutions. In 2020 43rd International Convention on Information, Communication and Electronic Technology (MIPRO) (pp. 419-424). IEEE.

[11] Moens, P., Bracke, V., Soete, C., Vanden Hautte, S., Nieves Avendano, D., Ooijevaar, T., ... \& Van Hoecke, S. (2020). Scalable Fleet Monitoring and Visualization 
Journal of Information Technology and Digital World (2021)

Vol. 03/ No. 01

Pages: $18-28$

https://www.irojournals.com/itdw/

DOI: https://doi.org/10.36548/jitdw.2021.1.003

for Smart Machine Maintenance and Industrial IoT Applications. Sensors, 20(15), 4308.

[12] Korhonen, N. (2020). Managing and Optimising IoT Data and ML applications dependencies.

[13] Pham, T. V., Tran, N. N. Q., Pham, H. M., Nguyen, T. M., \& Ta Minh, T. (2020, November). Efficient Low-Latency Dynamic Licensing for Deep Neural Network Deployment on Edge Devices. In 2020 The 3rd International Conference on Computational Intelligence and Intelligent Systems (pp. 44-49).

[14] Smys, S. (2020). A Survey on Internet of Things (IoT) based Smart Systems. Journal of ISMAC, 2(04), 181-189.

[15] Bashar, D. A. (2020). Review on sustainable green Internet of Things and its application. J. Sustain. Wireless Syst, 1(4), 256-264. 